\title{
Discrimination between Gamma and Log-Normal Distributions by Ratio of Minimized Kullback-Leibler Divergence
}

\author{
Ali-Akbar Bromideh \\ Hamdelan Koodak Foundation \\ Tehran, Iran \\ bromideh@gmail.com \\ Reza Valizadeh \\ Department of Statistics \\ Faculty of Mathematics \\ Yazd University, Yazd, Iran
}

\begin{abstract}
The Gamma and Log-Normal distributions are frequently used in reliability to analyze lifetime data. The two distributions overlap in many cases and make it difficult to choose the best one. The ratio of maximized likelihood (RML) has been extensively used in choosing between them. But the KullbackLeibler information is a measure of uncertainty between two functions, hence in this paper, we examine the use of Kullback-Leibler Divergence (KLD) in discriminating either the Gamma or Log-Normal distribution. Therefore, the ration of minimized Kullback-Leibler Divergence (RMKLD) test statistic is introduced and its applicability will be explained by two real data sets. Although the consistency of the new test statistic with RML is convinced, but the KLD has higher probability of correct selection when the null hypothesis is Gamma.
\end{abstract}

Keywords: Gamma Distribution, Kullback-Leibler Divergence, Log-Normal Distribution, Model Discrimination, Probability of Correct Selection, Ratio of Maximized Likelihood.

\section{Introduction}

Generally, positively skewed data play an important role in the reliability analysis. There are some well-known statistical distributions to model such data. For instance, Weibull, Inverse Gaussian, Gamma and Log-Normal distributions have been used in analyzing skewed positive data. These distributions are often interchangeable and commonly used to model certain lifetimes in reliability and survival analysis (Wiens, 1999). Although these models may provide similar data fit for moderate sample sizes, still it is desirable to select the correct model and make the best possible decision based on observed data. Often choosing a particular model is difficult and the relevant effect of mis-selection of model can be quite severe (Kundu and Manglick, 2005).

Gamma distribution has been used frequently in reliability analysis to describe the distribution and behaviour of lifetime data. Characteristics and details on this distribution has been widely studied by Cohen and Whitten (1988) and Burnham and Anderson (2002). On the other hand, Log-Normal distribution is commonly used to model lifetimes in reliability and survival analysis, among several other distributions. However, survival times of patients with certain types of cancer, failure times of semiconductor devices, and insurance claim payments are a few of examples where can be well modeled by either 
distributions. See Meeker and Escobar (1998), Blishke and Murthy (2000) and Crow and Shimitzu (1988)and the references therein for an overview on the two models.

Selection of the best model among several potential and candidate distributions is a prime problem of interest in lifetime analysis (Lawless, 1982). It is well-known that Gamma and Log-Normal distributions can be used quite effectively to analyze positive skewed data sets (Wiens, 1999). It is not easy to discriminate between the two distributions because they are quite flexible with possible overlapping behaviors in some cases. The problem of discrimination between the two distributions for testing whether some given data follow one of them is not a new subject in statistics and it has been well studied in the literature, so far. For instance, see (Dumonceaux and Antle, 1973; Kundu and Manglick, 2004; Pascual, 2005; Pasha et. al., 2006; and Dey and Kundu, 2009). The idea has been examined by discriminating between Gamma and Weibull distributions (Bain and Englehardt, 1980; Fearn and Nebenzahl, 1991; and Mohd Saat et al., 2008), between Gamma and Log-Normal distributions (Firth, 1988; Wiens, 1999; Kundu and Manglick, 2005). The subject has been extended to select between Log-Normal and Weibull (and also further other potential distributions) and discriminating more than two distributions (Dumonceaux and Antle, 1973; Dey and Kundu, 2009; Basu et al., 2009; Dey and Kundu, 2012; Bromideh, 2012).

So far, the ratio of maximized likelihood (RML) is mostly used in the literature to choose suitable models among others. But in some cases it is not the best test statistic and definitely it relays on sample size. For instance, to discriminate between Weibull and Log-Normal, RML is not a suitable selection criteria due to lack of inclusion of the location parameter of Log-Normal $(\mu)$ and on the other hand, for small sample size it has low power (Dumonceaux and Antle, 1973). Mohd Saat et al (2008) compared RML with Vuong's closeness test to discriminant between Gamma and Weibull, in which they found both methods relatively similar. Similar work to compare RML with KolmogorovSmirnov and Chi-Squared (with asymptomatic properties) has been studied and some inconsistency among them are reported (Basu et al, 2009). Despite of significant amount of work on discrimination by different methods, there is no much work to use the KLD and compare it with alternatives test statistic (Bromideh, 2012). However, this paper aims to fill this gap by examining selection between Gamma and Log-Normal distributions.

Due to increasing applications of lifetime models, special attention is given to the discrimination between Gamma and Log-Normal distributions. Therefore, we consider the problem of discriminating between the two distributions. Further, this paper aims to introduce a new test statistic based on Kullback-Leibler information (distance/divergence) for model selection purposes. An advantage of this method is that it incorporates information contained in both models. Second, all parameters of both models play important role in the testing statistic. Generally, the KLD between two models ( $\mathbf{P}$ and $\mathbf{Q}$ ) indicates that it can be used to determine "how far away" a probability distribution $\mathbf{P}$ (say, Gamma) from another distribution $\mathbf{Q}$, (say Log-Normal) (Burnham and Anderson, 2002). Recently, Bromideh (2012) introduced a new test statistics based on KLD to discriminate between two models. Applicability of his approach needs to generate critical values and then judge to reject or accept the null hypothesis. In this paper we extended his method by elimination of such calculation and make the algorithm 
very simple to test the hypotheses. In fact, we proposed the ratio of minimized KLD (RMKLD) as a test statistic, and hence, there is no need to generate critical values, which is given in the following sections.

The rest of the paper is organized as follows. In the following section, a brief presentation of Gamma and Log-Normal distributions will be provided. In section 3, the proposed test statistic (RMKLD) will be discussed and the simulated probability of correct selection (PCS) for different parameters and sample sizes will be reported. Two real data sets are analyzed in section 4 to explain how the proposed method works in practice and their PCS will be reported. Finally, we will conclude the discussion in the last section.

\section{Preliminary: Gamma and Log-Normal Distributions}

Gamma and Log-Normal distributions are among the possible models to analysis lifetime data in reliability and survival analysis. Both distributions are well-known and their properties have been comprehensively discussed in statistical literature (Burnham and Anderson, 2002); but the notation is somehow different. To make it clear, the Gamma and Log-Normal distributions are recalled.

A positive random variable $X$ is said to has a Gamma distribution, denoted by $G a(\alpha, \beta)$, when it has the probability density function (PDF) of

$$
h(x \mid \alpha, \beta)=\frac{x^{\alpha-1}}{\beta^{\alpha}} \exp \left(-\left(\frac{x}{\beta}\right)^{\alpha}\right)
$$

where $x>0$, and $\alpha>0, \beta>0$ are shape and scale parameters, respectively. The MLE of $\alpha$ and $\beta$ are calculated by:

$$
\hat{\beta}=\left(\frac{\sum_{i=1}^{n} x_{i}}{n}\right)^{\hat{\alpha}}
$$

A numerical analysis is required to estimate the unknown parameters of $\alpha$ and $\beta$ in a Gamma distribution. The developed $\mathrm{R}$ codes to estimate the parameters of a Gamma distribution can be shared upon request.

A random variable $X$ is distributed as $\log$-Normal, denoted as $L N\left(\mu, \sigma^{2}\right)$, if $\ln (X)$ is Normally distributed, e.g. $\ln (X) \sim N\left(\mu, \sigma^{2}\right)$. The PDF of $X$ is given by:

$$
g\left(x \mid \mu, \sigma^{2}\right)=\frac{1}{\sqrt{2 \pi} \sigma x} \exp \left(-\frac{(\ln (x)-\mu)^{2}}{2 \sigma^{2}}\right)
$$

where $x>0, \mu>0$ and $\sigma>0$. The MLE of $\mu$ and $\sigma^{2}$ are given below, respectively:

$$
\hat{\mu}=\frac{1}{n} \sum_{i=1}^{n} \ln \left(X_{i}\right) \text { and } \hat{\sigma}^{2}=\frac{1}{n} \sum_{i=1}^{n}\left(\ln \left(X_{i}\right)-\hat{\mu}\right)^{2} .
$$




\section{Model Selection: Gamma or Log-Normal?}

Let $x_{1}, x_{2}, \ldots, x_{k}$ are independent and identically distributed (iid) random variables from any one of the two probability distributions. Consider testing these hypotheses:

$$
H_{0}: X_{i} \sim G a(\alpha, \beta)
$$

against

$$
H_{1}: X_{i} \sim L N\left(\mu, \sigma^{2}\right)
$$

The main purpose of this paper is testing (5) against (6). Among the various testing methods, the most attention has been taken into consideration by goodness-of-fitness and RML. Initially, Dumonceaux and Antle (1973) introduced a test statistic with the concept of ratio of two likelihoods. Previously, a simplified version of RML (as the ratio of Loglogarithm of two distributions) is widely used (Kundu and Manglick, 2005; Pascual, 2005; Mohd Saat et al, 2008). We recall the RML for testing (5) against (6) as:

$$
T=\ln \left[\frac{L_{G a}(\hat{\alpha}, \hat{\beta})}{L_{L N}(\hat{\mu}, \hat{\sigma})}\right]
$$

where $L_{L N}$ and $L_{G a}$ denotes on likelihoods of Log-Normal and Gamma distributions, respectively (see Kundu and Manglick, 2005). Recently, Bromideh (2012) introduced KLD as a new test statistic to discriminate between Weibull and Log-Normal distributions, but it relies on generated critical values to test the hypotheses. To make it easy to follow, we extended his method by replacing the KLD with RMKLD which is presented in the following subsection.

\section{Kullback-Leibler Divergence based Test Statistic}

In probability and information theory, the Kullback-Leibler divergence (also information discrepancy, information gain, and relative entropy) is a non-symmetric measure of the difference (dissimilarity) between two probability distributions $\mathbf{f}$ and $\mathbf{h}$. The KullbackLeibler information between two models $\mathbf{f}$ and $\mathbf{h}$ is defined for continuous functions as:

$$
K L D(f, h)=\int f(x) \ln \left(\frac{f(x)}{h(x)}\right) d x
$$

It denotes the "information lost when $\mathbf{h}$ is used to approximate $\mathbf{f}$ or the distance from $\mathbf{h}$ to f." In other words, the KLD is a measure of inefficiency of assuming that the distribution is $\mathbf{h}$ when the true distribution is $\mathbf{f}$. Since the measure from $\mathbf{f}$ to $\mathbf{h}$ is not the same as the measure from $\mathbf{h}$ to $\mathbf{f}$, then it can be conceptualized as a "directed/oriented distance" between the two models (Burnham and Anderson, 2002).

The KLD is a natural distance function between models and it is a fundamental quantity in science and information theory. Also, it is a logical base for model selection in conjunction with likelihood inference. Values of KLD are not based on only the mean and variance of the distributions; rather, the distributions in their entirety are the subject 
of comparison. The later is regarded an advantage of the KLD as a test statistic. It is well known that $K L D(h, f) \neq K L D(f, h)$ and $K L D(f, h) \geq 0$ and the equality holds if and only if $f=h$ (Burnham and Anderson, 2002). The smaller $K L D(f, h)$ means that "f" is preferred."

However, the KLD (based test statistic) is considered as a ruler to measure the similarity between the two hypotheses/distributions. We define our test statistic as the natural logarithm of two ratios of KLDs. The idea is similar to RML, in which we are interested in selection of a model maximizing the likelihood. But in the new test statistic, we are interested to select the model minimizing the KLD, that's why we named it as ratio of minimized KLD (RMKLD). To simplify the concept, we introduce the RMKLD testing procedure as follows: 1

- Calculate $\operatorname{KLD}(\mathrm{f}, \mathrm{h})$ and $\operatorname{KLD}(\mathrm{h}, \mathrm{f})$

- Compute $K L D=\ln \left[\frac{K L D(f, h)}{K L D(h, f)}\right]$

- Select $\mathbf{f}$ if $K L D<0$.

In the following we provide KLD calculation for Gamma and Log-Normal distributions.

\section{Kullback-Leibler Divergence of Gamma and Log-Normal}

The KLD between a Log-Normal and Gamma distributions is defined as:

$$
\begin{array}{rlrl}
K L D\left(h_{L N}(x), f_{G a}(x)\right) & = & \int_{0}^{\infty} h_{L N}(x) \ln \left(\frac{h_{L N}(x)}{f_{G a}(x)}\right) d x \\
& =H\left(h_{L N}(x)\right)-\int_{0}^{\infty} h_{L N}(x) \ln \left(f_{G a}(x)\right) d x
\end{array}
$$

where $f_{G a}(x)$ denotes on Gamma distribution and $h_{L N}(x)$ is the PDF of Log-Normal distribution and $H\left(h_{L N}(x)\right)$ is the Entropy of Log-Normal distribution.

Finally, the KLD test statistic for testing Log-Normality vs. Gamma is given by:

$$
\begin{array}{r}
K L D\left(h_{L N}(x), f_{G a}(x)\right)=\ln (\Gamma(\alpha))+\alpha(\ln (\beta)-\mu)-\frac{1}{2} \ln \left(2 \pi e \sigma^{2}\right) \\
+\frac{1}{\beta} \exp \left(\mu+\frac{\sigma^{2}}{2}\right)
\end{array}
$$

However, small values of $K L D$ in (10), indicates that the data come from a Log-Normal distribution (Burnham and Anderson, 2002). As we are considering the choice of a model as a test of hypothesis, it is important to allow either of the models to be the null hypothesis. We suppose that the researcher would assign to the null hypothesis the model which prefers to use, unless there is convincing evidence that one should use the other. In order to allow the researcher this choice, we next provide the KLD for testing a Gamma 
vs. Log-Normal distribution. In other words, the KLD for testing $H_{0}: x \sim G a(\alpha, \beta)$ vs. $H_{1}: x \sim L N\left(\mu, \sigma^{2}\right)$ is given by:

$$
\begin{aligned}
K L D\left(f_{G a}(x), h_{L N}(x)\right)= & \ln (\sqrt{2 \pi} \sigma)-\ln (\Gamma(\alpha))-\alpha(1-\Psi(0, \alpha)) \\
& +\frac{1}{2 \sigma^{2}}\left\{[\mu-\ln (\beta)-\Psi(\alpha)]^{2}+\Psi(1, \alpha)\right\}
\end{aligned}
$$

where $\Psi(n, \alpha)$ is the $n^{\text {th }}$ polygamma function (i.e, the $n^{\text {th }}$ derivative of the digamma function). Again, the smaller values of (Error! Reference source not found.) emphasize on Gamma distribution.

Finally, the selection procedure is: "Choose the Log-Normal distribution if $\ln \left\{\frac{K L D\left(h_{L N}(x), f_{G a}(x)\right.}{K L D\left(f_{G a}(x), h_{L N}(x)\right.}\right\}<0$; otherwise choose the Gamma distribution as the best model." Since it is difficult to compute the exact distributions of $K L D\left(h_{L N}(x), f_{G a}(x)\right)$ and $K L D\left(f_{G a}(x), h_{L N}(x)\right)$, therefore, we use Mote Carlo simulations to compute the PCS for different sample sizes and parameters. Detail of simulation is provided in the following section.

\section{Simulation: PCS of RMKLD}

In this section we explain how the proposed RMKLD test statistic works for different parameters and sample sizes. The Monte Carlo simulations are used to compute the probability of correct selection for both KLD tests provided in (10) and (11). We consider different sample sizes $(n=20,30,50,100)$ and different parameters of the null distributions. In the previous similar studies, it's found that $\mu$ and $\beta$ have a negligible impact on RML and by simulation we found the same results in our KLD approach. We, therefore, set them to be equal to 1 .

First, we consider the case of (5) when the distribution of null hypothesis is Gamma. For this case, we set $\alpha=(0.5,1.0,1.5,2.0)$, and generate a random sample of size $\mathbf{n}$ from a $G a(\alpha, 1)$. We compute $K L D\left(f_{G a}(x), h_{L N}(x)\right)$ and $K L D\left(h_{L N}(x), f_{G a}(x)\right)$ from the equation (10) and (11), respectively. We, therefore, replicate the process 15,000 times and compute the percentage of the times if $K L D<0$ as the PCS for Gamma distribution. We have also computed the PCS for RML method and the results are reported in Table 1.

\begin{tabular}{|l|l|l|l|l|l|}
\hline & $\alpha \downarrow n \rightarrow$ & 20 & 30 & 50 & 100 \\
\hline & 0.5 & 0.9531 & 0.9815 & 0.9967 & 1.0000 \\
\hline & 1.0 & 0.8447 & 0.9005 & 0.9576 & 0.9922 \\
\hline RMKLD & 1.5 & 0.7793 & 0.8359 & 0.9067 & 0.9709 \\
\hline & 2.0 & 0.7405 & 0.7948 & 0.8667 & 0.9439 \\
\hline & 0.5 & 0.8589 & 0.9151 & 0.9647 & 0.9955 \\
\hline & 1.0 & 0.7763 & 0.8341 & 0.9103 & 0.9725 \\
\hline$R M L$ & 1.5 & 0.7321 & 0.787 & 0.8637 & 0.9451 \\
\hline & 2.0 & 0.7052 & 0.7605 & 0.8326 & 0.9159 \\
\hline
\end{tabular}


The PCS simulated by Monte Carlo (with 15,000 iteration) when the null distribution is Gamma.

From Table 1, we understand that KLD works better than the RML, because it generates higher PCS (about 4-10\%) in particular for small sample size. In other words, the error type I for KLD is remarkably less, compared to the RML one. For instance, consider the $G a(0.5,1)$. The PCS for KLD is $95.31 \%$ and $85.89 \%$ for RML, which indicates the error type I ( $\alpha$ ) equals to 4.69 for KLD and 14.11 for RML. It is also found that both methods behave similarly, for example, as sample size increases the PCS captures higher values, as expected. Moreover, as shape parameter $(\alpha)$ increases, the PCS decreases.

Second, we consider the case of (6); in which the null hypothesis denotes on LogNormality and the alternative is Gamma. In this case, we set $\sigma=(0.5,0.8,1.0,1.5)$ and generate a random sample of size $\mathbf{n}$ from $L N\left(1, \sigma^{2}\right)$. We compute $K L D\left(h_{L N}(x), f_{G a}(x)\right)$, $K L D\left(f_{G a}(x), h_{L N}(x)\right)$, and $R M K L D$, as the logarithm of the ratio of the two KLDs. We, therefore, replicate the process 15,000 times and compute the percentage of the cases if $R M K L D<0$ as the PCS for Log-Normal. Results are reported in Table 2, in which we also reported the PCS for RML test for comparison purposes.

\begin{tabular}{|l|l|l|l|l|l|}
\hline & $\sigma \downarrow n \rightarrow$ & 20 & 30 & 50 & 100 \\
\hline & 0.5 & 0.6255 & 0.6619 & 0.7225 & 0.8061 \\
\hline & 0.8 & 0.6701 & 0.7179 & 0.7843 & 0.8815 \\
\hline RMKLD & 1.1 & 0.6761 & 0.7333 & 0.8107 & 0.9023 \\
\hline & 1.5 & 0.6433 & 0.7157 & 0.7994 & 0.9009 \\
\hline & 0.5 & 0.6263 & 0.6749 & 0.7384 & 0.8241 \\
\hline & 0.8 & 0.7087 & 0.7611 & 0.8291 & 0.9247 \\
\hline RML & 1.1 & 0.7604 & 0.8197 & 0.8935 & 0.9645 \\
\hline & 1.5 & 0.7993 & 0.8665 & 0.9349 & 0.9873 \\
\hline
\end{tabular}

The PCS simulated by Monte Carlo (15,000 iteration) when the null distribution is LogNormal.

From the Table 2, RMKLD performs same to RML for small $\sigma$, but as $\sigma$ increases PCS for KLD decreases which is in contrast with RML test. This is due to the fact that as variance of a Log-Normal increases, its shape sharply concentrates on the right side and it gets far from Gamma distribution shape. Also, the RML is highly affected by $\sigma$. However, the error type I for RMKLD is almost higher than RML, especially for $\sigma>0.5$.

\section{Data Analysis: Implementation of the RMKLD Test}

To illustrate the use and applicability of the RMKLD test statistic, we analyze two real life-data sets in which a selection between Gamma and Log-Normal is of a prime interest. 
Data 1. We consider the following observations from Mohd Saat et al (2008) in which they tried to test whether the data come from a Gamma or a Log-Normal distribution. The data are the average duration of Hypopnea in seconds for 25 subjects of Obstructive Sleep Apnea (OSA): 14.7, 17.8, 16.5, 17.7, 28.5, 18.1, 32.2, 27.6, 22.3, 31.8, 22.0, 23.1, $31.6,18.4,28.3,16.5,21.8,23.7,27.6,17.2,20.0,20.6,19.0,18.7,19.2$.

For the data at hand, we have: $\hat{\alpha}=19.325, \hat{\beta}=1.148$ (for Gamma distribution); $\hat{\mu}=3.074$, and $\hat{\sigma}=0.226$ for Log-Normal distribution. The data were initially analyzed by Mohd Saat et al (2008) for discriminating between Weibull and Gamma distributions. By RML test, they reported that the data come from a Gamma distribution. But when compared between Gamma and Log-Normality, we found that Log-Normal fits well. So, by using the RMKLD, we decided to test the data if they fit well with a Gamma or a LogNormal distribution.

We have $K L D\left(h_{L N}(x), f_{G a}(x)\right)=0.004358$ and $K L D\left(f_{G a}(x), h_{L N}(x)\right)=0.004755$, and then, $K L D=\ln \left[\frac{K L D\left(h_{L N}(x), f_{G a}(x)\right)}{K L D\left(f_{G a}(x), h_{L N}(x)\right)}\right]=-0.0872<0 ;$ and consequently, we can not reject the Log-Normality. The PCS for RMKLD and RML in this case is the same at the value about $58 \%$.

Again the Log-Normality is confirmed and both test statistics are consistent. The fitted Gamma and Log-Normal distributions along with the original data are shown in Figure 1. The figure shows that it's difficult to discriminant between the two distributions due to their very close fitness and overlapping.

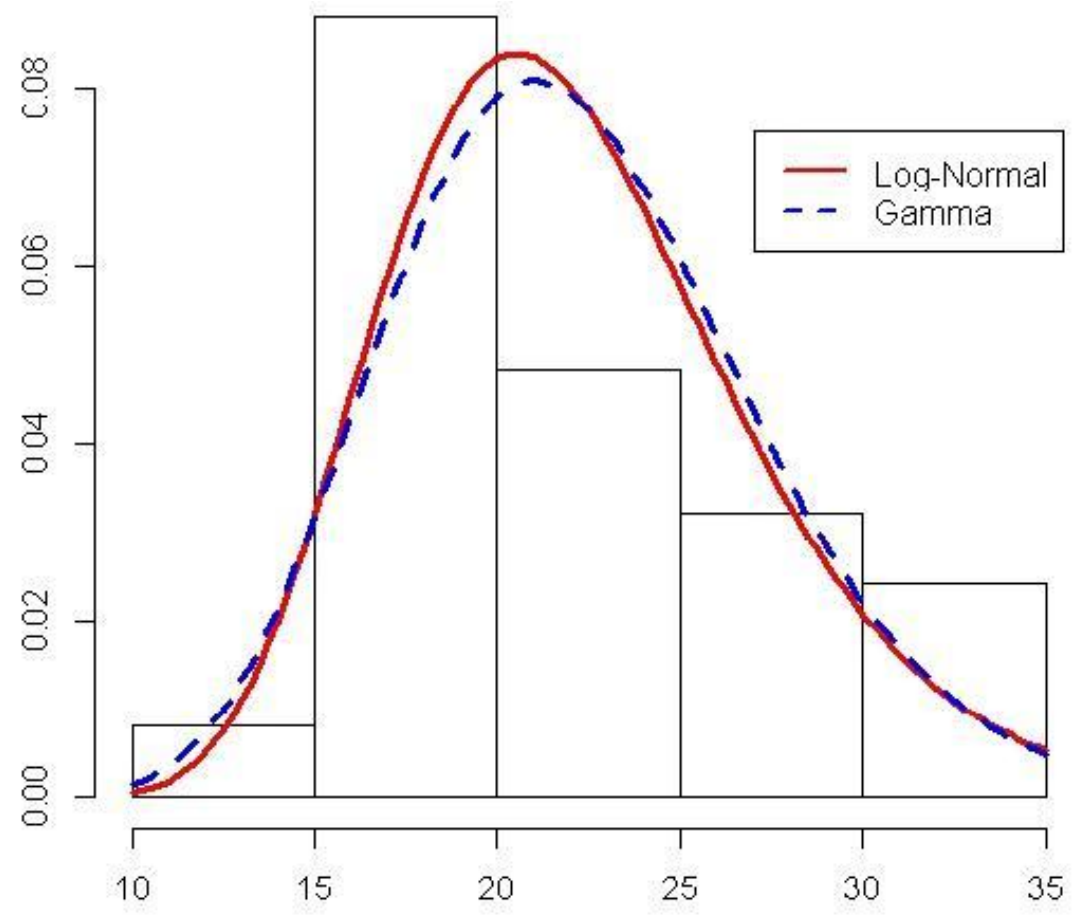

Figure 1: The histogram of data 1 and the two fitted distribution functions. 
Data 2. Suppose the following observations (as given by Lieblein and Zelen (1956) for the lifetime) are used to test whether the data come from a Gamma or a Log-Normal. The data given arose in tests on endurance of deep groove ball bearings. The data are the number of million revolutions before failure for each of the lifetime tests and they are: $17.88,28.92$, 33.00, 41.52, 42.12, 45.60, 48.80, 51.84, 51.96, 54.12, 55.56, 67.80, 68.44, $68.64,68.88,84.12,93.12,98.64,105.12,105.84,127.92,128.04,173.40$.

For these data we obtain $\hat{\mu}=4.1506$ and $\hat{\sigma}=0.5215$ for Log-Normal distribution and $\hat{\alpha}=4.02873$ and $\hat{\beta}=17.92744$ for the Gamma model. By the RML test, Gamma model fits better than Log-Normal. Note that Kundu and Manglick (2005) accepted Log-Normal by a mistake (mis-calculation of log-Likelihood of Gamma)!

Again, from (10) and (11) we have $K L D\left(h_{L N}(x), f_{G a}(x)\right)=0.02288528$ and $K L D\left(f_{G a}(x), h_{L N}(x)\right)=0.02208866, \quad$ and $\quad$ then $\quad R M K L D=0.03542948>0 ; \quad$ and consequently, we reject the Log-Normality and conclude that Gamma fits better with data set 2. The PCS for RMKLD is $67 \%$ and for RML is $65 \%$ in this case. Since in this case, the PCS for Gamma is better than the Log-Normal, then accepting Gamma fitness generates the less error type I, with respect to Log-Normal distribution. Finally, the consistency of RMKLD with RML is confirmed. The Figure 2 visualize the fitted distributions on the data 2 and suitability of Gamma is convinced.

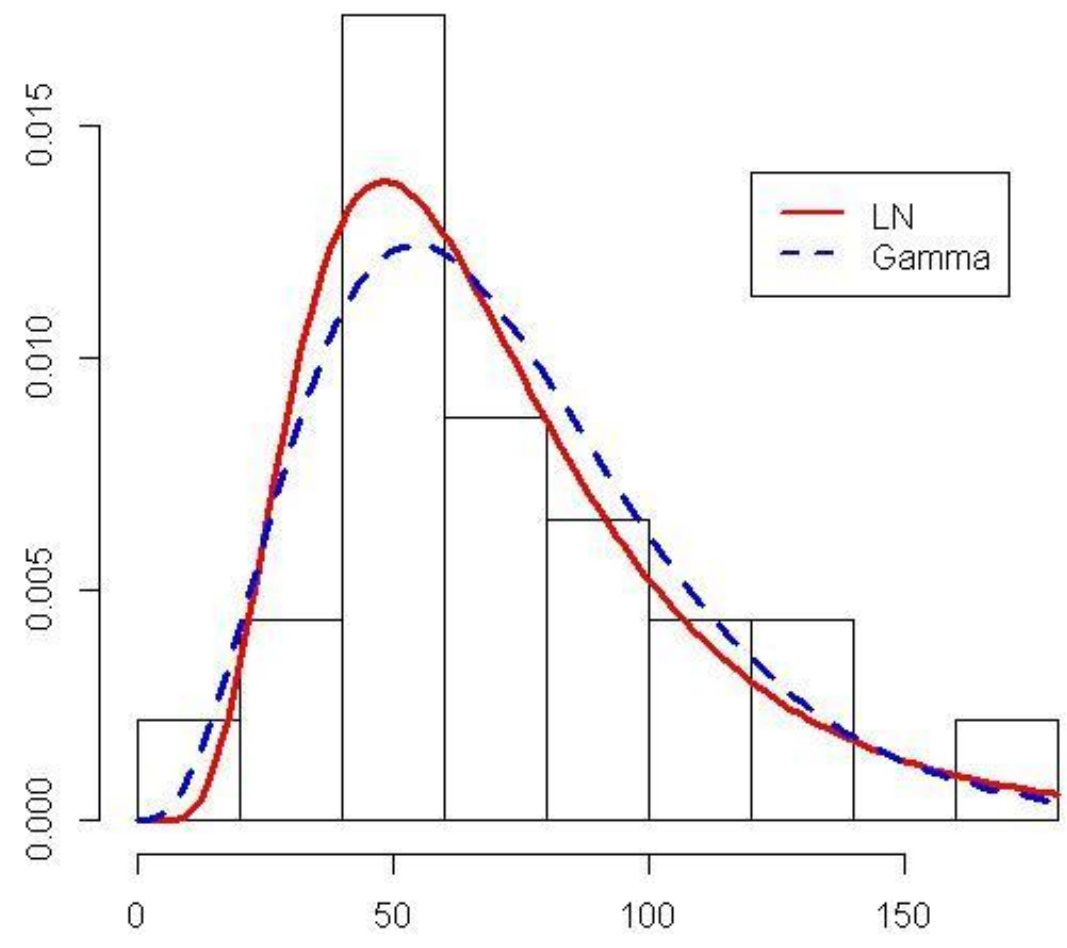

Figure 2: The histogram of data 2 and the two fitted distribution functions. 


\section{Conclusion}

In this paper we consider the problem of discriminating between two overlapping families of distribution functions, namely Log-Normal and Gamma. It is easy to realize the concept of Kullback-Leibler Divergence (information or distance) based test statistic and its usage in practice, but its application on discrimination of two known and overlapping distributions has less attention. The prime aim of this paper is to introduce a new test statistic, namely ratio of minimized KLD. It is observed that the proposed test statistic is consistent with alternative testing statistic, say RML. It is also found that the RMKLD works better than RML for small sample size when the null hypothesis is Gamma. On the other hand, when the null hypothesis is Log-Normal, it's cautious to use RMKLD. This behaviour needs more investigation from theoretical and simulation point of views to understand the main reason of such behaviours. Remind that the comparison of test statistics with each others is another story, which can cover in the future papers. Finally, it is suggested to interested research to test the approach for other similar distributions, such as (Generalized) Gamma, GE, Inverse Gaussian, ... and compare the result with RML and as well as other testing approaches. Finding the exact and/or asymptotic distribution of the proposed test statistic can be an interesting research topic in this regard.

\section{References}

1. Bain, L.J. and Engelhardt, M. (1980). "Probability of correct selection of Weibull versus gamma based on likelihood ratio," Comm. Statist. Ser. A. 9, 375-381.

2. Blishke, W.R. and Murthy, D.N.P. (2000). "Reliability modeling, prediction and optimization," Wiley Interscience Publications.

3. Bromideh, Ali-Akbar (2012). "Discriminating Between Weibull and Log-Normal Distributions Based on Kullback-Leibler Divergence", Istanbul University Econometrics and Statistics e-Journal, Vol. 16, Issue 1, pages 44-54.

4. Burnham, K. P., and Anderson, D. R. (2002). "Model selection and multimodel inference: a practical information-theoretic approach," 2nd eds., Springer, New York.

5. Cohen, A.C. and Whitten, B.J. (1988). "Parameter estimation in reliability and life span models," Marcel Dekker Inc, New York.

6. Crow, E.L. and Shimitzu, K. (1988). "Log-Normal Distributions, Theory and Applications, " Marcel Dekker Inc., New York.

7. Dey, A. K. and Kundu, D. (2009). "Discriminating among the Log-Normal, Weibull and Generalized Exponential distributions," IEEE Transactions on Reliability, vol. 58, no. 3, 416-424.

8. Dey, A. K. and Kundu, D. (2012). "Discriminating between the Weibull and Lognormal distributions for type-II censored data" Statistics ,vol. 46, no. 2, 197 $214,2012$. 
9. Dumonceaux, R., and Antle, C.E., (1973). "Discrimination between the lognormal and the Weibull distributions," Technometrics 15 (4), 923-926.

10. Fearn, D.H. and Nebenzahl, E. (1991). "On the maximum likelihood ratio method of deciding between the Weibull and Gamma distributions", Communications in Statistics - Theory and Methods, vol. 20, 579-593.

11. Firth, D. (1988). "Multiplicative errors: Log-Normal or Gamma?, Journal of the Royal Statistical Society, Ser. B, 2, 266-268.

12. Gross, A.J., and Clark, V.A. (1975). "Survival distribution: reliability application in the biomedical science," John Wiley \& Sons Inc.

13. Lawless, J.F., (1982). "Statistical Models and Methods for Lifetime Data," Wiley, New York.

14. Meeker and Escobar (1998). "Statistical Methods for Reliability Data," New York: John Wiley \& Sons Inc.

15. Mohd Saat, N. Z.; Jemain, A. A. and Al-Mashoor, S. H. (2008). "A Comparison of Weibull and Gamma Distributions in Application of Sleep Spnea," Asian Journal of Mathematics and Statistics, 1 (3), 132-138.

16. Kundu, D. and Manglick, A. (2004). "Discriminating between the Weibull and Log-Normal distributions", Naval Research Logistics, vol. 51, 893-905.

17. Kundu, D. and Manglick, A. (2005). "Discriminating between the Log-Normal and gamma distributions", Journal of the Applied Statistical Sciences, vol. 14, 175-187, 2005.

18. Pascual, F.G. (2005). "Maximum likelihood estimation under misspecied LogNormal and Weibull distributions", Communications in Statistics - Simulation and Computations vol. 34, No. 3, 503 - 524.

19. Pasha, G. R., Shuaib Khan, M. and Pasha, Ahmed Hesham, (2006). "Discrimination Between Weibull and Log-Normal Distributions For Lifetime data", Journal of Research (Science), Bahauddin Zakariya University, Multan, Pakistan. Vol. 17, No.2, April, pp. 103-114.

20. Wiens, B.L. (1999). "When Log-Normal and Gamma modles give different results: a case study," The American Statistician, 53, 2, 89-93. 